\title{
A review on exosome-based cancer therapy
}

\author{
Alex Von Schulze, Fengyan Deng \\ Department of Molecular and Integrative Physiology, University of Kansas Medical Center, Kansas City, KS 66160, USA. \\ Correspondence to: Dr. Fengyan Deng, Department of Molecular and Integrative Physiology, University of Kansas Medical \\ Center, Kansas City, KS 66160, USA. E-mail: fdeng@kumc.edu
}

How to cite this article: Schulze AV, Deng F. A review on exosome-based cancer therapy. J Cancer Metastasis Treat 2020;6:42. http://dx.doi.org/10.20517/2394-4722.2020.79

Received: 6 Aug 2020 First Decision: 21 Sep 2020 Revised: 18 Oct 2020 Accepted: 27 Oct 2020 Published: 6 Nov 2020

Academic Editor: Robert J Griffin Copy Editor: Cai-Hong Wang Production Editor: Jing Yu

\begin{abstract}
Cancer is one of the leading causes of mortality and morbidity globally. Many types of cancer treatments have been developed, such as chemotherapy, surgery, radiotherapy, and immunotherapy. However, these therapies can also kill healthy cells and lead to severe side effects. Therefore, scientists are looking for new strategies to eliminate cancerous cells specifically. Exosomes, nanometer-sized lipid bilayer-enclosed vesicles secreted from various cell types, exist in nearly all body fluids, including blood, breast milk, saliva, urine, bile, pancreatic juice, cerebrospinal, and peritoneal fluids. They carry myriad donor cell-derived bioactive molecules such as proteins, lipids, and RNAs (including microRNA and IncRNA) and can deliver them to both nearby and distant recipient cells. Due to these characteristics, exosomes have attracted great interest in cancer treatment (especially serving as a biological carrier for some drugs, microRNA, IncRNA, inhibitors, and antibodies). In this paper, we will review the current knowledge of exosome therapeutic applications in cancer.
\end{abstract}

Keywords: Exosomes, cancer, cancer therapy, gene carrier, drug carrier

\section{INTRODUCTION}

Cancer remains the leading cause of death globally. There are over 200 types of cancer which claim more than 10 million lives annually ${ }^{[1]}$. Despite a lot of research focusing on this grave disease, cancer therapeutics still have the lowest clinical trial success rate of all major diseases. This is likely due to the fact that it is hard for our immune system to distinguish cancerous cells from healthy cells. For instance, current therapeutics like radiotherapy and chemotherapy not only kill the cancerous cells, but also healthy cells ${ }^{[1]}$. Therefore, developing new therapeutic strategies in order to precisely eliminate cancerous cells is an urgent need.

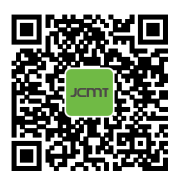


Exosomes are nanosized extracellular vesicles which are secreted by various cells throughout the body ${ }^{[2-5]}$. They carry important bioactive molecules including proteins, lipids, DNA (e.g., mitochondrial DNA and genomic DNA), and RNAs [e.g., microRNAs (miRNAs), long non coding RNAs (lncRNAs), and messenger RNA (mRNA)], and can transfer them to the recipient cells - thus playing a crucial role in cellcell communication ${ }^{[2-6]}$. Exosomes are present in most bodily fluids including blood, breast milk, saliva, urine, bile, pancreatic juice, cerebrospinal, and peritoneal fluids ${ }^{[2-6]}$. Through the circulating flow, they can transfer from their original cells to distant tissues where they localize to target cells by binding their surface molecules to receptors on the surface of the target cells ${ }^{[2-6]}$. Due to the advantage of their unique biocompatibility and high stability, exosomes have attracted great interest in cancer treatment as an effective anti-cancer drug delivery carrier or gene carrier. In this review, we provide an overview for studies of exosomes application in cancer therapies, as well as their advantages and challenges.

\section{DUAL ROLES OF EXOSOMES IN TUMORIGENESIS}

According to the exosome database ExoCarta (www.exocarta.org), 9,769 proteins, 3,408 mRNA, 2,838 miRNA, and 1,116 lipids have been identified in exosomes from different organisms and bodily fluids ${ }^{[7]}$. By transferring bioactive molecules from the donor cells to the recipient cells, exosomes play crucial roles in cell-cell communication ${ }^{[2-5]}$. Many studies have shown that cancer cells release a larger number of exosomes to exchange information with other cells both nearby and at distance ${ }^{[8-10]}$. Through delivering their bioactive cargoes (including proteins, miRNAs, and lncRNAs), cancer cell-derived exosomes contribute to the formation of the pre-metastatic microenvironment, tumor growth and progression, immune escape, angiogenesis, anti-apoptotic signaling, drug-resistance, and so on ${ }^{[8-10]}$ [Figure 1]. Meanwhile, exosomes from healthy cells, such as dendritic cells (DCs), B cells, and T cells, play an important role in inhibiting tumor growth $^{[11-14]}$ [Figure 1]. To date, numerous miRNAs, lncRNAs, and proteins have been found to play important roles in cancer progression [Table 1]. Therefore, depending on their cell of origin and their bioactive cargo, exosomes can play dual roles in cancer regulation, either inhibiting or promoting growth.

\section{EXOSOME-BASED CANCER THERAPIES}

Since exosomes can unload bioactive cargo to cancer cells, they have attracted great interest in cancer treatment ${ }^{[6-72]}$. Currently, several different methods have been developed for cancer therapies: (1) using naturally derived exosomes from immune cells to suppress cancer cells ${ }^{[66]} ;(2)$ inhibiting the release of cancer-derived exosomes; (3) using exosomes as gene carriers ${ }^{[69]}$; and (4) using exosomes as anti-cancer drug carriers ${ }^{[6,71]}$ [Figure 2].

\section{Naturally derived exosomes for cancer therapy}

In cancer-immunity, DCs are involved in the first step of tumor cell growth inhibition by capturing neoantigens and triggering the tumor-specific cytotoxic lymphocyte response ${ }^{[73]}$. DC-derived exosomes (Dex) contain various bioactive cargoes responsible for antigen presentation, making them ideal for the treatment of cancer ${ }^{[74,75]}$. In 1998 , Zitvogel et al ${ }^{[76]}$ found that tumor peptide-pulsed Dex were able to activate the antigen-specific cytotoxic T lymphocytes response in vivo and eradicate or suppress growth of established murine tumors in a T cell-dependent manner. Moreover, Munich et al. ${ }^{[77]}$ found that Dex can directly kill tumor cells and activate naturel killer (NK) cells through TNF superfamily ligands. It has also been found that cancer cell-derived exosomes have an immunostimulatory effect on anti-tumor DCs ${ }^{[6]]}$. Thus, Dex represent an important strategy for cancer therapy.

\section{Interfering with cancer cell-derived exosomes for cancer therapy}

Cancer cell-derived exosomes are considered to accelerate cancer pathogenesis by contributing to the formation of the pre-metastatic microenvironment, tumor growth and progression, immune escape, angiogenesis, anti-apoptotic signaling, and drug-resistance ${ }^{[8-10]}$. Therefore, inhibition of the cancer cell- 


\section{Dural role of exosomes in cancer progression}

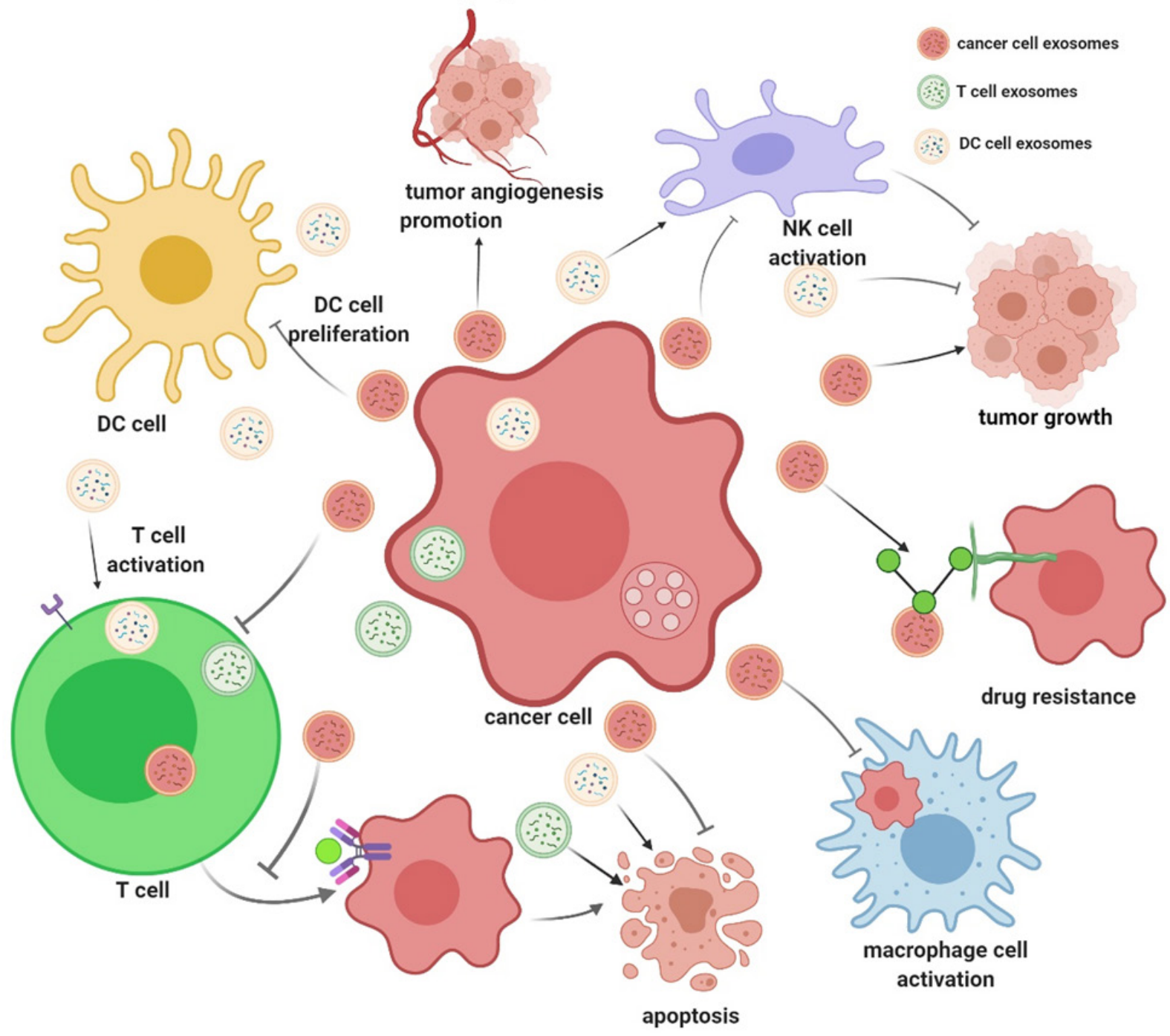

Figure 1. Dual role of exosomes in cancer progression. Cancer cell-derived exosomes contribute to the formation of the pre-metastatic microenvironment, tumor growth and progression, immune escape, angiogenesis, anti-apoptotic signaling, drug-resistance, and so on. While exosomes from healthy cells including dendritic cells, B cells, and T cells, play crucial role in inhibiting tumor growth (This figure was created with BioRender.com)

derived exosomes synthesis, release, and uptake may serve as an effective cancer therapy ${ }^{[78]}$. With the use of a mouse model, Bobrie et al. ${ }^{[78]}$ found that blockade of Rab27a, a key mediator of exosome secretion, resulted in decreased primary tumor growth and lung dissemination of metastatic carcinoma (4T1) cells. It was found that miR-494 is enriched in melanoma-derived exosomes, and that exosomal transport of miR-494 promotes metastasis ${ }^{[79]}$. More specifically, inhibiting the function of Rab27a blocked exosomal transfer of miR-494 and resulted in inhibiting melanoma growth and metastasis ${ }^{[79]}$. Another possible way to mitigate tumor growth is to simple remove circulating exosomes via a hemofiltration system (such as the Aethlon $\mathrm{ADAPT}^{\mathrm{TM}}$ system). This alternative may also serve as another possible therapeutic approach for reversing immune dysfunction and improving the immune responses to tumor growth ${ }^{[80]}$. Finally, multiple studies have discovered that blocking exosomal uptake is another potentially effective method for cancer therapy ${ }^{[81,82]}$. For example, the heparan sulfate (HS) proteoglycans has been discovered as internalizing receptors of cancer cell-derived exosomes ${ }^{[82]}$. HSPG deficiency, or the HS mimetic (heparin) treatment, significantly reduced exosome uptake and exosome-mediated stimulation of cancer cell migration ${ }^{[82]}$. In all three cases (blocking exosomal secretion, exosomal removal, and blocking exosomal uptake), disrupting 
Table 1. List of exosomal bioactive molecules involved in various cancer progression

\begin{tabular}{|c|c|}
\hline Exosome cargos & Function in cancer progression \\
\hline \multicolumn{2}{|l|}{ MicroRNAs } \\
\hline miR-122 & Promotes metastasis ${ }^{[15]}$ \\
\hline $\operatorname{miR}-9$ & Promotes tumor angiogenesis ${ }^{[16]}$ \\
\hline miR-105 & Induces vascular leakiness and promoting metastasis ${ }^{[17]}$ \\
\hline miR-135b & Enhances angiogenesis ${ }^{[18]}$ \\
\hline miR-93-5P & Promotes the proliferation of esophageal cancer cells $s^{[19]}$ \\
\hline miR-132 & Promotes angiogenesis ${ }^{[20]}$ \\
\hline miR-23 & Enhances angiogenesis and vascular permeability ${ }^{[21]}$ \\
\hline miR-200 & Promotes cancer cell metastasis ${ }^{[22]}$ \\
\hline miR-126 & Promotes the anti-tumor response $\mathrm{e}^{[23]}$ \\
\hline miR-92a & Regulates hepatic pre-metastatic niche in lung cancer ${ }^{[24]}$ \\
\hline miR-210 & Increases angiogenesis and tumor progression ${ }^{[25]}$ \\
\hline $\operatorname{miR}-221$ & Promotes oncogenic activity in gastric cancer ${ }^{[26]}$ \\
\hline miR-141 & Associates with the presence of cancer ${ }^{[27]}$ \\
\hline $\operatorname{miR}-375$ & Associates with the presence of cancer ${ }^{[27]}$ \\
\hline miR-494 & Enhances angiogenesis through the suppression of PTEN and the Akt/eNOS pathway ${ }^{[28,29]}$ \\
\hline miR-21 & Promotes hepatocellular carcinoma growth ${ }^{[30]}$ \\
\hline miR-103 & Promotes tumor progression and angiogenesis ${ }^{[31]}$ \\
\hline miR-542-3p & Regulates tumor growth/Wnt signaling through targeting cdh17 and TRAF4 ${ }^{[29]}$ \\
\hline miR-19a & Metastatic colonization through specifically silencing PTEN ${ }^{[32]}$ \\
\hline miR-92 & Metastatic colonization through specifically silencing PTEN ${ }^{[32]}$ \\
\hline $\operatorname{miR}-17$ & Metastatic colonization through specifically silencing PTEN ${ }^{[32]}$ \\
\hline $\operatorname{miR}-373$ & Raises the invasion and metastasis of tumor ${ }^{[33]}$ \\
\hline miR-501 & confers doxorubicin resistance and tumorigenesis ${ }^{[34]}$ \\
\hline miR-1247-3p & Promotes cancer progression ${ }^{[35]}$ \\
\hline hsa-miR-940 & Induces extensive osteoblastic lesions in the resulting tumors ${ }^{[36]}$ \\
\hline \multicolumn{2}{|l|}{ Long noncoding RNAs } \\
\hline InCRNA-HOTTIP & Promotes cisplatin resistance $e^{[37]}$ \\
\hline IncRNA-ZFAS1 & Enhances gastric cancer cell proliferation and migration ${ }^{[38]}$ \\
\hline LncRNAs-MALAT-1 & Promotes cell proliferation and migration in non-small cell lung cancer ${ }^{[39]}$ \\
\hline IncRNA-TUC339 & Promotes cell proliferation, clonogenic growth and tumor growth ${ }^{[40]}$ \\
\hline LncRNA-PART1 & Induces gefitinib resistance ${ }^{[44]}$ \\
\hline LncRNA-UCA1 & Enhances UCA1 expression and resistance to cetuximab ${ }^{[42]}$; Induce tamoxifen resistance ${ }^{[43]}$ \\
\hline IncRNA-Sox2ot & Promotes EMT and stemness ${ }^{[44]}$ \\
\hline lincRNA-ROR & Suppresses cell apoptosis ${ }^{[45]}$ \\
\hline IncRNA-AFAP1-AS1 & Induces resistance to trastuzumab ${ }^{[46]}$ \\
\hline IncRNA-ARSR & Induces resistance to the sunitinib ${ }^{[47]}$ \\
\hline IncRNA-SNHG14 & Induces resistance to trastuzumab ${ }^{[48]}$ \\
\hline InCRNA PCSEAT & Enhances cellular proliferation and motility ${ }^{[49]}$ \\
\hline IncRNA-SBF2-AS1 & Enhances temozolomide resistance ${ }^{[50]}$ \\
\hline IncRNA-H19 & Enhances resistance of doxorubicin ${ }^{[51]}$ \\
\hline IncRNA-RP11838N2.4 & Promotes erlotinib resistance ${ }^{[52]}$ \\
\hline \multicolumn{2}{|l|}{ Proteins } \\
\hline MIF & Initiates pro-metastatic effect ${ }^{[53]}$ \\
\hline EGFR vIII & Activates the VEGF signaling ${ }^{[54]}$ \\
\hline TGF- $\beta$ & Suppresses T cell proliferation ${ }^{[55]}$ \\
\hline ANXA6 & Facilitates the establishment of lung metastasis ${ }^{[56]}$ \\
\hline Integrins & Promotes cancer progression ${ }^{[57]}$ \\
\hline Tspan8 & Essential for the crosstalk between cancer initiating cells and their surrounding ${ }^{[58]}$ \\
\hline CD151 & Essential for the crosstalk between cancer initiating cells and their surrounding ${ }^{[58]}$ \\
\hline MICA & Prevents NK cell-mediated tumor elimination ${ }^{[59]}$ \\
\hline TNF- $\alpha$ & Induces immune cells death ${ }^{[60]}$ \\
\hline CEMIP & Promotes cancer cell colonization in brain metastasis ${ }^{[61]}$ \\
\hline PD-L1 & Inhibits immune responses ${ }^{[62,63]}$, promotes tumor growth ${ }^{[64,65]}$ \\
\hline
\end{tabular}

PTEN: phosphatase and tensin homolog; EMT: epithelial-mesenchymal transition; VEGF: vascular endothelial growth factor; TNF- $\alpha$ : tumor necrosis factor- $\alpha$; TGF- $\beta$ : transforming growth factor- $\beta$; PD-L1: programmed death-ligand 1; EGFR: epidermal growth factor receptor 


\section{Exosome-Based Cancer Therapies}

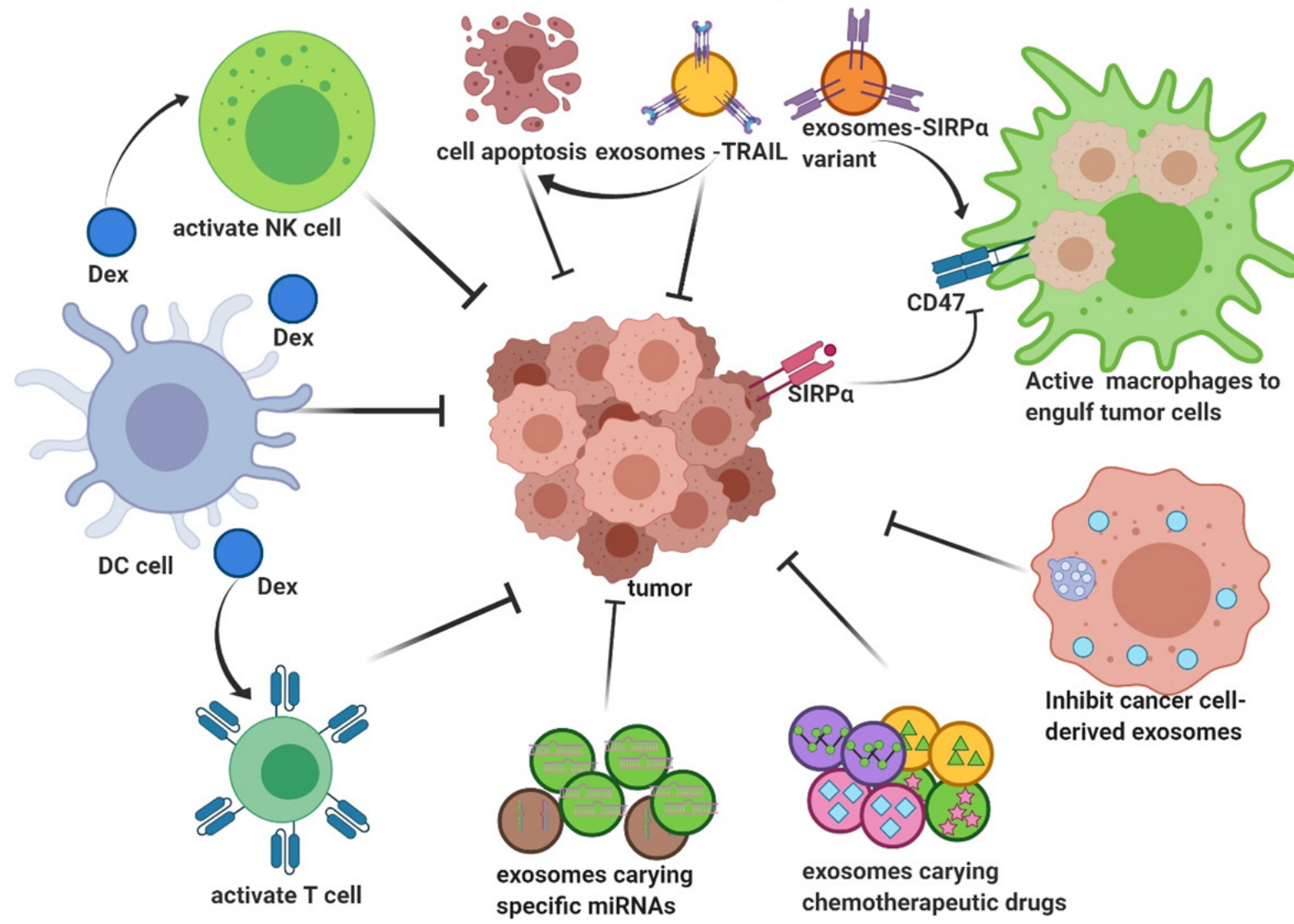

Figure 2. Exosome-based cancer therapies. Currently, four different methods have been developed for cancer therapies: (1) natural exosomes from some immune cells to suppress cancer cells; (2) inhibition of cancer cells-derived exosomes; (3) exosomes as gene carriers; and (4) exosomes as anti-cancer drug carriers (This figure was created with BioRender.com)

cell-cell communication from cancer-cell derived exosomes appears to offer an exciting new way to treat cancer.

\section{Exosomes as gene carriers for cancer therapy}

Although, there is great potential for exosomes in cancer therapy, the use of natural exosomes is hard and rarely achieves the expected therapeutic result. Fortunately, engineered exosomes carrying specific proteins, RNAs, or drugs have been found to possess great potential for effective cancer treatment.

\section{Exosomes as miRNA carrier for cancer therapy}

miRNAs are endogenous, small, non-coding RNAs that can regulate gene expression by binding to target mRNAs. Therefore, miRNAs could be a powerful tool for cancer therapy. However, miRNAs are easily degraded in vivo and delivery of miRNAs to their specific target cells/tissue/organ is a major challenge. As exosomes are stable small vesicles that can carry functional bioactive molecules long distances with a high degree of target specificity ${ }^{[2-6]}$, they have been suggested as a potential carrier for miRNAs in cancer therapy. Over the last few years, many scientists have focused on exosomal-based delivery of miRNAs and miRNA inhibitors for cancer therapy ${ }^{[83-88]}$. For instance, in 2013, Katakowski et al ${ }^{[83]}$ reported that exosomes enriched with the anti-glioma miRNA (miRNA-146b) can suppress glioma growth in vitro and can also significantly reduce glioma xenograft growth in rats. Similarly, miR-101-enriched exosomes can suppress osteosarcoma cell invasion/migration in vitro and suppress metastasis in vivo ${ }^{[84]}$. Wang et al ${ }^{[85]}$ also reported that exosomes loaded with miR-335-5p can decrease cancer growth and invasion both in vitro and in vivo. 
Interestingly, O'Brien et al. ${ }^{[86]}$ used engineered mesenchymal stem cells to secrete exosomes enriched with miR-379 for in vivo therapy of breast cancer. Importantly, they found that systemic administration of miR-379 enriched exosomes can significantly reduce tumor activity. Another miRNA, miR-145-5p, was also found to inhibit pancreatic ductal adenocarcinoma cell proliferation and invasion, as well as increased apoptosis. Moreover, exosomes transfected with miR-145-5p were able to inhibit pancreatic ductal adenocarcinoma cell proliferation and invasion through TGF- $\beta / S \operatorname{Smad} 3$ pathways ${ }^{[87]}$. On the other hand, inhibitors of exosomal miRNAs, which play key roles in cancer progression through exosome, have become another effective method for cancer therapy. For example, exosomal miR-25-3p is a metastasispromoting miRNA of colorectal cancer. Exosomes enriched with miR-25-3p dramatically promoted vascular permeability and colorectal cancer metastasis in mice liver and lung. Nevertheless, these effects can be rescued by blockage of exosomal miR-25-3p by a miR-25-3p inhibitor ${ }^{[88]}$.

\section{Exosomes as protein carrier for cancer therapy}

Recently, many scientists have begun to develop an exosomal-based cancer vaccine ${ }^{[89-91]}$. For example, TNF-alpha-related-apoptosis-inducing-ligand (TRAIL), a cytokine, functions as a ligand that induces cell apoptosis ${ }^{[92,93]}$. Rivoltini et al. ${ }^{[94]}$ reported that TRAIL-armed exosomes could promote apoptosis in cancer cells and control tumor progression in vivo. Furthermore, IL-18 enriched exosomes enhance Th1 cytokine release and proliferation of peripheral blood mononuclear cells, suggesting that IL-18 enriched exosomes harbor more capability to induce specific anti-tumor immunity as they trigger a bigger immune response ${ }^{[95]}$. Yang et al. ${ }^{[96]}$ also found that IL-2 enriched exosomes induce the antigen-specific Th1-polarized immune response and cytotoxic $\mathrm{T}$ lymphocyte response more efficiently, leading a significant inhibition of tumor growth in mice. Exosomes can also be used as carriers of protein antagonists. For example, signal regulatory protein $\alpha(\operatorname{SIRP} \alpha)$ can interact with $\mathrm{CD} 47$, a "don't eat me" signal that limits the ability of macrophages to engulf tumor cells. Exosomes carrying SIRP $\alpha$ antagonists could significantly increase tumor phagocytosis, as has been observed in tumor-bearing mice ${ }^{[97]}$.

\section{Exosomes as chemotherapeutic drug carriers for cancer therapy}

Anti-tumor chemotherapeutic drugs can effectively kill fast-growing tumor cells. However, these drugs can also kill the normal, healthy cells that are fast-growing, causing serious side effects. Besides, for some hydrophobic drugs, it is a challenge for them to target tumor cells with any kind of specificity. Therefore, an effective carrier for these drugs is badly needed. Due to their naturally derived origin and their stable lipid bilayer structure, exosomes have the great potential to serve as an effective carrier for chemotherapeutic agents. As early as 2012, Tang et al ${ }^{[98]}$ reported that tumor cell-derived microparticles can be used as chemotherapeutic drug carriers. They found that chemotherapeutic drugs loaded onto microparticles had a potent anti-tumor effect both in vitro and in vivo ${ }^{[98]}$. In 2015, Kim et al ${ }^{[99]}$ developed a exosome-based formulation of paclitaxel (PTX), a commonly used chemotherapeutic agent, to overcome multiple drug resistance (MDR) in cancer cells. Three methods including incubation at room temperature, electroporation, and mild sonication were used to incorporate PTX into exosomes; among which, the mild sonication method resulted in the highest loading efficiency and sustained drug release ${ }^{[99]}$. PTX-loaded exosomes (exoPTX) increased cytotoxicity more than 50 times in drug resistant $\mathrm{MDCK}_{\mathrm{MDR} 1}(\mathrm{Pgp}+)$ cells $^{[99]}$. Furthermore, through a similar in vivo mice model, Kim et al ${ }^{[99]}$ found that exoPTX can efficiently target cancer cells and produce strong antineoplastic effects in mice with lung metastases. Similarly, Saari et al..$^{100]}$ also found that delivery of PTX via cancer cell-derived exosomes enhances the cytotoxicity of PTX in autologous prostate cancer cells. Furthermore, by modifying the exosome surface proteins, exosomes can deliver chemotherapeutic drugs to cancer cells with a high degree of specificity. For example, Tian et al. ${ }^{[101]}$ engineered Lamp2b-iRGD peptide ( $\alpha \mathrm{v}$ integrin-specific) expressing mouse immature DCs (imDCs), isolated their exosomes and used them to deliver doxorubicin (Dox). Using this approach, they found that iRGD-exosomes can efficiently target and deliver Dox to $\alpha \mathrm{v}$ integrin-positive breast cancer cells in vitro, and specifically to tumor tissues, resulting in inhibition of tumor growth in vivo ${ }^{[101]}$. 


\section{CONCLUSION AND FUTURE DIRECTIONS}

Due to their role in cancer progression and biological features, exosomes possess promising potential for cancer therapy. To date, numerous exosomes-based cancer therapies have been studied and developed including applying naturally derived immune cell exosomes to suppress cancer cells, inhibiting cancer cells-derived exosomal activity, and using exosomes as gene/drug carriers. However, there are considerable challenges to be solved. First, the difference among exosomes from different sources is still not clear. Second, the exosome number to get a therapeutic effect may be significantly different among different cancers. Third, scalability and heterogeneity of tumor may influence therapeutic outcome. Moreover, different functions of exosomes derived from different sources are not fully studied. Furthermore, knowledge on how exosomes can be modified so that they possess a high degree of specificity to particular cancer cells remains unclear. Finally, the storage and the stability of exosomes remains ill-defined. With these challenges, there is a vital need to systematically characterize exosomes derived from different cells/ tissue to choose the most efficient cells for specific cancer therapy. There is a further need to identify the specific cancer cell surface markers for designing exosomes as drug carriers with high specificity to cancer cells. Moreover, because of the complexity and the heterogeneity of the tumor, exosome-based cancer may need to combine with other approaches. Finally, clinical trials of exosome-based cancer therapy are urgently needed to determine the efficacy of the application. While there are still challenges ahead, it is clear that exosomes offer novel and important applications for the treatment of cancer.

\section{DECLARATIONS}

\section{Acknowledgments}

All figures were created with BioRender.com.

\section{Authors' contributions}

Conceived the general idea of the review, made up the structure and searched the literature: Deng F

Drafted the manuscript: Schulze AV, Deng F

\section{Availability of data and materials}

Not applicable.

\section{Financial support and sponsorship}

None.

\section{Conflicts of interest}

All authors declared that there are no conflicts of interest.

\section{Ethical approval and consent to participate}

Not applicable.

\section{Consent for publication}

Not applicable.

\section{Copyright}

(c) The Author(s) 2020.

\section{REFERENCES}

1. Carter S, Thurston DE. Immuno-oncology agents for cancer therapy. Pharmaceut J 2020; doi: 10.1211/PJ.2020.20207825

2. Daßler-Plenker J, Küttner V, Egeblad M. Communication in tiny packages: exosomes as means of tumor-stroma communication. Biochim Biophys Acta Rev Cancer 2020;1873:188340. 
3. Hannafon BN, Ding WQ. Intercellular communication by exosome-derived microRNAs in cancer. Int J Mol Sci 2013;14:14240-69.

4. Deng F, Miller J. A review on protein markers of exosome from different bio-resources and the antibodies used for characterization. $J$ Histotechnol 2019;42:226-39.

5. Deng F, Magee N, Zhang Y. Decoding the role of extracellular vesicles in liver diseases. Liver Res 2017;1:147-55.

6. Bruschi M, Ravera S, Santucci L, et al. The human urinary exosome as a potential metabolic effector cargo. Expert Rev Proteomics 2015;12:425-32

7. Keerthikumar S, Chisanga D, Ariyaratne D, et al. ExoCarta: a web-based compendium of exosomal cargo. J Mol Biol 2016;428:688-92.

8. Osaki M, Okada F. Exosomes and their role in cancer progression. Yonago Acta Med 2019;62:182-90.

9. Sun W, Luo JD, Jiang H, Duan DD. Tumor exosomes: a double-edged sword in cancer therapy. Acta Pharmacol Sin 2018;39:534-41.

10. Dilsiz N. Role of exosomes and exosomal microRNAs in cancer. Future Sci OA 2020;6:FSO465.

11. Utsugi-kobukai S, Fujimaki H, Hotta C, Nakazawa M, Minami M. MHC class I-mediated exogenous antigen presentation by exosomes secreted from immature and mature bone marrow derived dendritic cells. Immunology Letters 2003;89:125-31.

12. Hao S, Bai O, Li F, Yuan J, Laferte S, Xiang J. Mature dendritic cells pulsed with exosomes stimulate efficient cytotoxic T-lymphocyte responses and antitumour immunity. Immunology 2007;120:90-102.

13. Pitt JM, Charrier M, Viaud S, et al. Dendritic cell-derived exosomes as immunotherapies in the fight against cancer. $J$ Immunol 2014;193:1006-11.

14. Admyre C, Johansson SM, Paulie S, Gabrielsson S. Direct exosome stimulation of peripheral human T cells detected by ELISPOT. Eur $J$ Immunol 2006;36:1772-81.

15. Fong MY, Zhou W, Liu L, et al. Breast-cancer-secreted miR-122 reprograms glucose metabolism in premetastatic niche to promote metastasis. Nat Cell Biol 2015;17:183-94.

16. Zhuang $\mathrm{G}, \mathrm{Wu} \mathrm{X}$, Jiang $\mathrm{Z}$, et al. Tumour-secreted miR-9 promotes endothelial cell migration and angiogenesis by activating the JAKSTAT pathway. EMBO J 2012;31:3513-23.

17. Zhou W, Fong MY, Min Y, et al. Cancer-secreted miR-105 destroys vascular endothelial barriers to promote metastasis. Cancer Cell 2014;25:501-15.

18. Umezu T, Tadokoro H, Azuma K, Yoshizawa S, Ohyashiki K, Ohyashiki JH. Exosomal miR-135b shed from hypoxic multiple myeloma cells enhances angiogenesis by targeting factor-inhibiting HIF-1. Blood 2014;124:3748-57.

19. Liu MX, Liao J, Xie M, et al. miR-93-5p transferred by exosomes promotes the proliferation of esophageal cancer cells via intercellular communication by targeting PTEN. Biomed Environ Sci 2018;31:171-85.

20. Ma T, Chen Y, Chen Y, et al. MicroRNA-132, delivered by mesenchymal stem cell-derived exosomes, promote angiogenesis in myocardial infarction. Stem Cells Int 2018;2018:3290372.

21. Hsu YL, Hung JY, Chang WA, et al. Hypoxic lung cancer-secreted exosomal miR-23a increased angiogenesis and vascular permeability by targeting prolyl hydroxylase and tight junction protein ZO-1. Oncogene 2017;36:4929-42.

22. Le MT, Hamar P, Guo C, et al. miR-200-containing extracellular vesicles promote breast cancer cell metastasis. J Clin Invest 2014; $124: 5109-28$.

23. Monaco F, Gaetani S, Alessandrini F, et al. Exosomal transfer of miR-126 promotes the anti-tumour response in malignant mesothelioma: Role of miR-126 in cancer-stroma communication. Cancer Lett 2019;463:27-36.

24. Hsu YL, Huang MS, Hung JY, et al. Bone-marrow-derived cell-released extracellular vesicle miR-92a regulates hepatic pre-metastatic niche in lung cancer. Oncogene 2020;39:739-53.

25. Cui H, Seubert B, Stahl E, et al. Tissue inhibitor of metalloproteinases-1 induces a pro-tumourigenic increase of miR-210 in lung adenocarcinoma cells and their exosomes. Oncogene 2015;34:3640-50.

26. Ma M, Chen S, Liu Z, et al. miRNA-221 of exosomes originating from bone marrow mesenchymal stem cells promotes oncogenic activity in gastric cancer. Onco Targets Ther 2017;10:4161-71.

27. Bryant RJ, Pawlowski T, Catto JW, et al. Changes in circulating microRNA levels associated with prostate cancer. Br J Cancer 2012;106:768-74.

28. Ingenito F, Roscigno G, Affinito A, et al. The role of exo-miRNAs in cancer: a focus on therapeutic and diagnostic applications. Int J Mol Sci 2019;20:4687.

29. Rana S, Malinowska K, Zöller M. Exosomal tumor microRNA modulates premetastatic organ cells. Neoplasia 2013;15:281-95.

30. Cao LQ, Yang XW, Chen YB, Zhang DW, Jiang XF, Xue P. Exosomal miR-21 regulates the TETs/PTENp1/PTEN pathway to promote hepatocellular carcinoma growth. Mol Cancer 2019;18:148.

31. Hsu YL, Hung JY, Chang WA, et al. Hypoxic lung-cancer-derived extracellular vesicle microRNA-103a increases the oncogenic effects of macrophages by targeting PTEN. Mol Ther 2018;26:568-81.

32. Zhang L, Zhang S, Yao J, et al. Microenvironment-induced PTEN loss by exosomal microRNA primes brain metastasis outgrowth. Nature 2015;527:100-4.

33. Liu Q, Peng F, Chen J. The role of exosomal microRNAs in the tumor microenvironment of breast cancer. Int J Mol Sci 2019;20:3884.

34. Liu X, Lu Y, Xu Y, et al. Exosomal transfer of miR-501 confers doxorubicin resistance and tumorigenesis via targeting of BLID in gastric cancer. Cancer Lett 2019;459:122-34.

35. Fang T, Lv H, Lv G, et al. Tumor-derived exosomal miR-1247-3p induces cancer-associated fibroblast activation to foster lung metastasis of liver cancer. Nat Commun 2018;9:191.

36. Hashimoto K, Ochi H, Sunamura S, et al. Cancer-secreted hsa-miR-940 induces an osteoblastic phenotype in the bone metastatic microenvironment via targeting ARHGAP1 and FAM134A. Proc Natl Acad Sci U S A 2018;115:2204-9. 
37. Wang J, Lv B, Su Y, Wang X, Bu J, Yao L. Exosome-mediated transfer of lncRNA HOTTIP promotes cisplatin resistance in gastric cancer cells by regulating HMGA1/miR-218 Axis. Onco Targets Ther 2019;12:11325-38.

38. Pan L, Liang W, Fu M, et al. Exosomes-mediated transfer of long noncoding RNA ZFAS1 promotes gastric cancer progression. $J$ Cancer Res Clin Oncol 2017;143:991-1004.

39. Zhang R, Xia Y, Wang Z, et al. Serum long non coding RNA MALAT-1 protected by exosomes is up-regulated and promotes cell proliferation and migration in non-small cell lung cancer. Biochem Biophys Res Commun 2017;490:406-14.

40. Kogure T, Yan IK, Lin WL, Patel T. Extracellular vesicle-mediated transfer of a novel long noncoding RNA TUC339: a mechanism of intercellular signaling in human hepatocellular cancer. Genes Cancer 2013;4:261-72.

41. Kang M, Ren M, Li Y, Fu Y, Deng M, Li C. Exosome-mediated transfer of lncRNA PART1 induces gefitinib resistance in esophageal squamous cell carcinoma via functioning as a competing endogenous RNA. J Exp Clin Cancer Res 2018;37:171.

42. Yang YN, Zhang R, Du JW, et al. Predictive role of UCA1-containing exosomes in cetuximab-resistant colorectal cancer. Cancer Cell Int 2018;18:164.

43. Xu CG, Yang MF, Ren YQ, Wu CH, Wang LQ. Exosomes mediated transfer of lncRNA UCA1 results in increased tamoxifen resistance in breast cancer cells. Eur Rev Med Pharmacol Sci 2016;20:4362-8.

44. Li Z, Jiang P, Li J, et al. Tumor-derived exosomal lnc-Sox2ot promotes EMT and stemness by acting as a ceRNA in pancreatic ductal adenocarcinoma. Oncogene 2018;37:3822-38.

45. Takahashi K, Yan IK, Kogure T, Haga H, Patel T. Extracellular vesicle-mediated transfer of long non-coding RNA ROR modulates chemosensitivity in human hepatocellular cancer. FEBS Open Bio 2014;4:458-67.

46. Han M, Gu Y, Lu P, et al. Exosome-mediated lncRNA AFAP1-AS1 promotes trastuzumab resistance through binding with AUF1 and activating ERBB2 translation. Mol Cancer 2020;19:26.

47. Qu L, Ding J, Chen C, et al. Exosome-transmitted lncARSR promotes sunitinib resistance in renal cancer by acting as a competing endogenous RNA. Cancer Cell 2016;29:653-68.

48. Dong H, Wang W, Chen R, et al. Exosome-mediated transfer of lncRNA-SNHG14 promotes trastuzumab chemoresistance in breast cancer. Int J Oncol 2018;53:1013-26.

49. Yang X, Wang L, Li R, et al. The long non-coding RNA PCSEAT exhibits an oncogenic property in prostate cancer and functions as a competing endogenous RNA that associates with EZH2. Biochem Biophys Res Commun 2018;502:262-8.

50. Zhang Z, Yin J, Lu C, Wei Y, Zeng A, You Y. Exosomal transfer of long non-coding RNA SBF2-AS1 enhances chemoresistance to temozolomide in glioblastoma. J Exp Clin Cancer Res 2019;38:166.

51. Wang X, Pei X, Guo G, et al. Exosome-mediated transfer of long noncoding RNA H19 induces doxorubicin resistance in breast cancer. $J$ Cell Physiol 2020;235:6896-904.

52. Zhang W, Cai X, Yu J, Lu X, Qian Q, Qian W. Exosome-mediated transfer of lncRNA RP11838N2.4 promotes erlotinib resistance in nonsmall cell lung cancer. Int J Oncol 2018;53:527-38.

53. Costa-Silva B, Aiello NM, Ocean AJ, et al. Pancreatic cancer exosomes initiate pre-metastatic niche formation in the liver. Nat Cell Biol $2015 ; 17: 816-26$.

54. Al-Nedawi K, Meehan B, Kerbel RS, Allison AC, Rak J. Endothelial expression of autocrine VEGF upon the uptake of tumor-derived microvesicles containing oncogenic EGFR. Proc Natl Acad Sci U S A 2009;106:3794-9.

55. Rong L, Li R, Li S, Luo R. Immunosuppression of breast cancer cells mediated by transforming growth factor-beta in exosomes from cancer cells. Oncol Lett 2016;11:500-4.

56. Keklikoglou I, Cianciaruso C, Güç E, et al. Chemotherapy elicits pro-metastatic extracellular vesicles in breast cancer models. Nat Cell Biol 2019;21:190-202.

57. Paolillo M, Schinelli S. Integrins and exosomes, a dangerous liaison in cancer progression. Cancers (Basel) 2017;9:95.

58. Yue S, Mu W, Erb U, Zöller M. The tetraspanins CD151 and Tspan8 are essential exosome components for the crosstalk between cancer initiating cells and their surrounding. Oncotarget 2015;6:2366-84.

59. Ashiru O, Boutet P, Fernández-Messina L, et al. Natural killer cell cytotoxicity is suppressed by exposure to the human NKG2D ligand MICA*008 that is shed by tumor cells in exosomes. Cancer Res 2010;70:481-9.

60. Andreola G, Rivoltini L, Castelli C, et al. Induction of lymphocyte apoptosis by tumor cell secretion of FasL-bearing microvesicles. $J$ Exp Med 2002;195:1303-16.

61. Rodrigues G, Hoshino A, Kenific CM, et al. Tumour exosomal CEMIP protein promotes cancer cell colonization in brain metastasis. Nat Cell Biol 2019;21:1403-12.

62. Choi SH, Bylykbashi E, Chatila ZK, et al. Combined adult neurogenesis and BDNF mimic exercise effects on cognition in an Alzheimer's mouse model. Science 2018;361:eaan8821.

63. Fan Y, Che X, Qu J, et al. Exosomal PD-L1 retains immunosuppressive activity and is associated with gastric cancer prognosis. Ann Surg Oncol 2019;26:3745-55.

64. Kim DH, Kim H, Choi YJ, et al. Exosomal PD-L1 promotes tumor growth through immune escape in non-small cell lung cancer. Exp Mol Med 2019;51:1-13.

65. Yang Y, Li CW, Chan LC, et al. Exosomal PD-L1 harbors active defense function to suppress T cell killing of breast cancer cells and promote tumor growth. Cell Res 2018;28:862-4.

66. Liu H, Chen L, Peng Y, et al. Dendritic cells loaded with tumor derived exosomes for cancer immunotherapy. Oncotarget 2018;9:2887-94.

67. Gilligan KE, Dwyer RM. Engineering exosomes for cancer therapy. Int J Mol Sci 2017;18:1122.

68. Gomari H, Forouzandeh Moghadam M, Soleimani M. Targeted cancer therapy using engineered exosome as a natural drug delivery 
vehicle. Onco Targets Ther 2018;11:5753-62.

69. Lin Q, Qu M, Zhou B, et al. Exosome-like nanoplatform modified with targeting ligand improves anti-cancer and anti-inflammation effects of imperialine. J Control Release 2019;311-312:104-16.

70. Nie W, Wu G, Zhang J, et al. Responsive exosome nano-bioconjugates for synergistic cancer therapy. Angew Chem Int Ed Engl 2020;59:2018-22.

71. Pullan JE, Confeld MI, Osborn JK, Kim J, Sarkar K, Mallik S. Exosomes as drug carriers for cancer therapy. Mol Pharm 2019;16:1789-98.

72. Wang J, Zheng Y, Zhao M. Exosome-based cancer therapy: implication for targeting cancer stem cells. Front Pharmacol 2016;7:533.

73. Chen DS, Mellman I. Oncology meets immunology: the cancer-immunity cycle. Immunity 2013;39:1-10.

74. Pitt JM, André F, Amigorena S, et al. Dendritic cell-derived exosomes for cancer therapy. J Clin Invest 2016;126:1224-32.

75. Markov O, Oshchepkova A, Mironova N. Immunotherapy based on dendritic cell-targeted/-derived extracellular vesicles-a novel strategy for enhancement of the anti-tumor immune response. Front Pharmacol 2019;10:1152.

76. Zitvogel L, Regnault A, Lozier A, et al. Eradication of established murine tumors using a novel cell-free vaccine: dendritic cell-derived exosomes. Nat Med 1998;4:594-600.

77. Munich S, Sobo-Vujanovic A, Buchser WJ, Beer-Stolz D, Vujanovic NL. Dendritic cell exosomes directly kill tumor cells and activate natural killer cells via TNF superfamily ligands. Oncoimmunology 2012;1:1074-83.

78. Bobrie A, Krumeich S, Reyal F, et al. Rab27a supports exosome-dependent and -independent mechanisms that modify the tumor microenvironment and can promote tumor progression. Cancer Res 2012;72:4920-30.

79. Li J, Chen J, Wang S, et al. Blockage of transferred exosome-shuttled miR-494 inhibits melanoma growth and metastasis. J Cell Physiol 2019:15763-74.

80. Marleau AM, Chen CS, Joyce JA, Tullis RH. Exosome removal as a therapeutic adjuvant in cancer. $J$ Transl Med 2012;10:134.

81. Mulcahy LA, Pink RC, Carter DR. Routes and mechanisms of extracellular vesicle uptake. J Extracell Vesicles 2014;3:24641.

82. Christianson HC, Svensson KJ, van Kuppevelt TH, Li JP, Belting M. Cancer cell exosomes depend on cell-surface heparan sulfate proteoglycans for their internalization and functional activity. Proc Natl Acad Sci U S A 2013;110:17380-5.

83. Katakowski M, Buller B, Zheng X, et al. Exosomes from marrow stromal cells expressing miR-146b inhibit glioma growth. Cancer Lett 2013;335:201-4.

84. Zhang K, Dong C, Chen M, et al. Extracellular vesicle-mediated delivery of miR-101 inhibits lung metastasis in osteosarcoma. Theranostics 2020;10:411-25.

85. Wang F, Li L, Piontek K, Sakaguchi M, Selaru FM. Exosome miR-335 as a novel therapeutic strategy in hepatocellular carcinoma. Hepatology 2018;67:940-54.

86. O'Brien KP, Khan S, Gilligan KE, et al. Employing mesenchymal stem cells to support tumor-targeted delivery of extracellular vesicle (EV)-encapsulated microRNA-379. Oncogene 2018;37:2137-49.

87. Ding Y, Cao F, Sun H, et al. Exosomes derived from human umbilical cord mesenchymal stromal cells deliver exogenous miR-145-5p to inhibit pancreatic ductal adenocarcinoma progression. Cancer Lett 2019;442:351-61.

88. Zeng Z, Li Y, Pan Y, et al. Cancer-derived exosomal miR-25-3p promotes pre-metastatic niche formation by inducing vascular permeability and angiogenesis. Nat Commun 2018;9:5395.

89. Rountree RB, Mandl SJ, Nachtwey JM, et al. Exosome targeting of tumor antigens expressed by cancer vaccines can improve antigen immunogenicity and therapeutic efficacy. Cancer Res 2011;71:5235-44.

90. André F, Chaput N, Schartz NE, et al. Exosomes as potent cell-free peptide-based vaccine. I. Dendritic cell-derived exosomes transfer functional MHC class I/peptide complexes to dendritic cells. J Immunol 2004;172:2126-36.

91. Chaput N, Schartz NE, André F, et al. Exosomes as potent cell-free peptide-based vaccine. II. Exosomes in CpG adjuvants efficiently prime naive Tc1 lymphocytes leading to tumor rejection. J Immunol 2004;172:2137-46.

92. Wiley SR, Schooley K, Smolak PJ, et al. Identification and characterization of a new member of the TNF family that induces apoptosis. Immunity 1995;3:673-82.

93. Pitti RM, Marsters SA, Ruppert S, Donahue CJ, Moore A, Ashkenazi A. Induction of apoptosis by Apo-2 ligand, a new member of the tumor necrosis factor cytokine family. J Biol Chem 1996;271:12687-90.

94. Rivoltini L, Chiodoni C, Squarcina P, et al. TNF-related apoptosis-inducing ligand (TRAIL)-armed exosomes deliver proapoptotic signals to tumor site. Clin Cancer Res 2016;22:3499-512.

95. Dai S, Zhou X, Wang B, et al. Enhanced induction of dendritic cell maturation and HLA-A*0201-restricted CEA-specific CD8(+) CTL response by exosomes derived from IL-18 gene-modified CEA-positive tumor cells. J Mol Med (Berl) 2006;84:1067-76.

96. Yang Y, Xiu F, Cai Z, et al. Increased induction of antitumor response by exosomes derived from interleukin-2 gene-modified tumor cells. J Cancer Res Clin Oncol 2007;133:389-99.

97. Koh E, Lee EJ, Nam GH, et al. Exosome-SIRPa, a CD47 blockade increases cancer cell phagocytosis. Biomaterials 2017;121:121-9.

98. Tang K, Zhang Y, Zhang H, et al. Delivery of chemotherapeutic drugs in tumour cell-derived microparticles. Nat Commun 2012;3:1282.

99. Kim MS, Haney MJ, Zhao Y, et al. Development of exosome-encapsulated paclitaxel to overcome MDR in cancer cells. Nanomedicine 2016;12:655-64.

100. Saari H, Lázaro-Ibáñez E, Viitala T, Vuorimaa-Laukkanen E, Siljander P, Yliperttula M. Microvesicle- and exosome-mediated drug delivery enhances the cytotoxicity of Paclitaxel in autologous prostate cancer cells. J Control Release 2015;220:727-37.

101. Tian Y, Li S, Song J, et al. A doxorubicin delivery platform using engineered natural membrane vesicle exosomes for targeted tumor therapy. Biomaterials 2014;35:2383-90. 